\title{
ON THE HARDY-TYPE \\ INTEGRAL OPERATORS IN BANACH FUNCTION SPACES
}

\author{
Elena Lomakina and Vladimir Stepanov
}

\begin{abstract}
Characterization of the mapping properties such as boundedness, compactness, measure of non-compactness and estimates of the approximation numbers of Hardy-type integral operators in Banach function spaces are given.
\end{abstract}

\section{Introduction}

Let $X$ and $Y$ be two Banach spaces of measurable functions defined on $\mathbf{R}^{+}$. We consider the Hardy-type integral operator $K: X \rightarrow Y$ given by

$$
K f(x)=\varphi(x) \int_{0}^{x} k(x, y) \psi(y) f(y) d y, \quad x>0
$$

where the real functions $\varphi(x)$ and $\psi(x)$ (weights) are measurable and finite almost everywhere on $\mathbf{R}^{+}$, and the kernel $k(x, y) \geq 0$, satisfies

$$
\begin{aligned}
D^{-1}(k(x, z)+k(z, y)) & \leq k(x, y) \\
& \leq D(k(x, z)+k(z, y)), \quad x \geq z \geq y \geq 0,
\end{aligned}
$$

Keywords. Banach function spaces, Hardy-type integral operator, compact operator, approximation number.

1991 Mathematics subject classifications: 46E30, 47B38, 47G60.

The research work of the both authors was partially supported by the INTAS project 94-881 and of the second author by the Centre de Recerca Matemàtica at Barcelona and by the RFFR grant 97-01-00604. 
where the constant $D \geq 1$ does not depend on $x, y, z$. Typical examples of such a kernel are $(x-y)^{\alpha}, \alpha \geq 0 ; \log ^{\beta}\left(\frac{x}{y}\right), \beta \geq 0$; or $\left(\int_{y}^{x} h(s) d s\right)^{\gamma}$, $\gamma \geq 0$ with nonnegative $h(s)$, and their various combinations. Introduced by R. Oinarov [13], [14] the condition (1.2) extends in some sense the well-known $\Delta_{2}$-condition for convex functions [10] used in [12], [18] for convolution operators and thus, (1.2) seems to be a balance point between generality of conditions imposed on a kernel and implicitness of a criterion for the boundedness of the Hardy-type operators. A survey of the mapping properties of operators (1.1) with Oinarov's kernel in Lebesgue and Lorentz spaces can be found in [19].

The paper is devoted to operators of the form (1.1) acting in Banach spaces of Lebesgue-measurable functions on $\mathbf{R}^{+}$(see Definition 1 below). Investigation in this area was recently initiated by E. Berezhnoi [2], [3], who, in particular, characterized weak-type estimates for the operator (1.1) with the kernel $k(x, y) \geq 0$ increasing with respect to the first variable and also strong estimates, when $k(x, y)=1$ and the spaces $X$ and $Y$ satisfy an $\ell$-condition (see Definition 3 below). E. Berezhnoi [3] has also obtained some necessary and/or sufficient conditions for the boundedness of operators (1.1) with restrictions on $k(x, y) \geq 0$, stronger than (1.2).

Sections 2 and 3 contain definitions and the statement of the main results and further comments, respectively. Our first result characterizes the boundedness of the operator (1.1) with kernel satisfying (1.2) in the spaces $X$ and $Y$ satisfying an $\ell$-condition (Theorem 1 ). This leads to a characterization of the compactness (Theorem 2) and measure of noncompactness (Theorem 3) of the operator. Upper and lower estimates for the behaviour of the approximation numbers of operators (1.1), when $k(x, y)=1$, are given in Theorems 6 and 7 . Sections 4 and 5 provide the proofs.

\section{Definitions}

Definition 1 [1]. A real normed linear space $X=\left\{f:\|f\|_{X}<\infty\right\}$ is called a Banach function space (BFP) if in addition to the usual norm axioms $\|f\|_{X}$ satisfies the following conditions:

(1) $\|f\|_{X}$ is defined for every Lebesgue-measurable function $f$ on $\mathbf{R}^{+}$, and $f \in X$ if, and only if, $\|f\|_{X}<\infty ;\|f\|_{X}=0$ if, and only if, $f=0$ almost everywhere (a.e.);

(2) $\|f\|_{X}=\||f|\|_{X}$ for all $f \in X$;

(3) if $0 \leq f \leq g$ a.e., then $\|f\|_{X} \leq\|g\|_{X}$; 
(4) if $0 \leq f_{n} \uparrow f$ a.e., then $\left\|f_{n}\right\|_{X} \uparrow\|f\|_{X}$;

(5) if mes $E<\infty$, then $\left\|\chi_{E}\right\|_{X}<\infty$;

(6) if mes $E<\infty$, then $\int_{E} f(x) d x \leq C_{E}\|f\|_{X}$.

Given a BFS $X$, its associate space $X^{\prime}$ is defined by

$$
X^{\prime}=\left\{g: \int_{0}^{\infty}|f g|<\infty \text { for all } f \in X\right\},
$$

and endowed with the associate norm

$$
\|g\|_{X^{\prime}}=\sup \left\{\int_{0}^{\infty}|f g|:\|f\|_{X} \leq 1\right\} .
$$

$X^{\prime}$ is also a Banach function space satisfying axioms (1)-(6) and, moreover, $X^{\prime}$ is a norm fundamental subspace of the dual space $X^{*}$, that is the equality

$$
\|f\|_{X}=\sup \left\{\int_{0}^{\infty}|f g|:\|g\|_{X^{\prime}} \leq 1\right\}
$$

holds for all $f \in X[\mathbf{1}]$.

The spaces $X, X^{\prime}$ are complete normed linear spaces and $X^{\prime \prime}=X[\mathbf{1}]$.

The Hölder inequality

$$
\left|\int_{0}^{\infty} f g\right| \leq\|f\|_{X}\|g\|_{X^{\prime}}
$$

holds for all $f \in X$ and $g \in X^{\prime}$ and is sharp in both directions on the strength of (2.1) and (2.2). The relationships (2.1) and (2.2) give rise to the following

Principle of duality. $T: X \rightarrow Y$ is a bounded linear operator, that is $\|T f\|_{Y} \leq C\|f\|_{X}$ for all $f \in X$ with a finite positive constant $C$, if and only if

(i) $\left\|T^{\prime} g\right\|_{X^{\prime}} \leq C\|g\|_{Y^{\prime}}$ for all $g \in Y^{\prime}$, where the conjugate operator $T^{\prime}: Y^{\prime} \rightarrow X^{\prime}$ is defined by the formulae

$$
\int_{0}^{\infty}(T f) g=\int_{0}^{\infty} f\left(T^{\prime} g\right)
$$

or

(ii) $\left|\int_{0}^{\infty}(T f) g\right| \leq C\|f\|_{X}\|g\|_{Y^{\prime}}$ for all $f \in X$ and $g \in Y^{\prime}$, with the same constant $C$. The least possible constant $C$ defines the norm $\|T\|$ and, thus $\|T\|_{X \rightarrow Y}=\left\|T^{\prime}\right\|_{Y^{\prime} \rightarrow X^{\prime}}$. 
$X$ has absolutely continuous norm (AC-norm), if for all $f \in X$, $\left\|f \chi_{E_{n}}\right\|_{X} \rightarrow 0$ for every sequence of sets $\left\{E_{n}\right\} \subset \mathbf{R}^{+}$such, that $\chi_{E_{n}}(x) \rightarrow 0$ a.e. We assume throughout the paper that $X^{\prime}$ and $Y$ have AC-norms.

Let $\ell$ be a Banach sequence space (BSS), what means that axioms (1)-(6) are satisfied with respect to the count measure and let $\left\{e_{k}\right\}$ denote the standard basis in $\ell$.

Definition 2 [3]. Given a BFS $X$ and a BSS $\ell, X$ is said to be $\ell$-concave, if for any sequence of disjoint intervals $\left\{J_{k}\right\}$ such that $\bigcup J_{k}=\mathbf{R}^{+}$, and for all $f \in X$

$$
\left\|\sum_{k} e_{k}\right\| \chi_{J_{k}} f\left\|_{X}\right\|_{\ell} \leq d_{1}\|f\|_{X},
$$

where $d_{1}$ is a finite positive constant independent of $f \in X$ and $\left\{J_{k}\right\}$. Analogously, BFS $Y$ is said to be $\ell$-convex, if for any sequence of disjoint intervals $\left\{I_{k}\right\}$, such that $\bigcup I_{k}=\mathbf{R}^{+}$and for all $g \in Y$

$$
\|g\|_{Y} \leq d_{2}\left\|\sum_{k} e_{k}\right\| \chi_{I_{k}} g\left\|_{Y}\right\|_{\ell}
$$

for a finite positive constant $d_{2}>0$, independent of $g \in Y$ and $\left\{I_{k}\right\}$.

Definition 3 [3]. (The Berezhnoi $\ell$-condition). We say, that Banach function spaces $X$ and $Y$ satisfy an $\ell$-condition, if there exists a Banach sequence space $\ell$ such that $X$ is $\ell$-concave and $Y$ is $\ell$-convex simultaneously.

Let $\ell^{\prime}$ denote the associate space. We need the following

Lemma 1 [3]. Let $Y$ be an $\ell$-convex BFS and suppose (2.4) holds. Then $Y^{\prime}$ is an $\ell^{\prime}$-concave BFS and

$$
\left\|\sum_{k} e_{k}\right\| \chi_{I_{k}} f\left\|_{Y^{\prime}}\right\|_{\ell^{\prime}} \leq d_{2}\|f\|_{Y^{\prime}},
$$

for all $f \in Y^{\prime}$ and $\left\{I_{k}\right\}$, such that $\bigcup I_{k}=\mathbf{R}^{+}$.

Throughout the paper the expressions of the form $0 \cdot \infty, 0 / 0, \infty / \infty$ are taken equal to zero, the inequality $A \ll B$ means $A \leq c B$, where $c$ depends only on $D$, and possibly on the constants $d_{1}$ and $d_{2}$ of Definition 2; however the relationship $A \approx B$ is interpreted as $A \ll B \ll A$ or $A=c B . \chi_{E}$ denotes the characteristic function (indicator) of a set $E \subset \mathbf{R}^{+}$. 


\section{Statement of the main results}

Put for all $t \geq 0$

$$
\begin{aligned}
& A_{0}=\sup _{t>0} A_{0}(t)=\sup _{t>0}\left\|\chi_{[t, \infty]} \varphi\right\|_{Y}\left\|\chi_{[0, t]}(\cdot) k(t, \cdot) \psi(\cdot)\right\|_{X^{\prime}}, \\
& A_{1}=\sup _{t>0} A_{1}(t)=\sup _{t>0}\left\|\chi_{[t, \infty]}(\cdot) k(\cdot, t) \varphi(\cdot)\right\|_{Y}\left\|\chi_{[0, t]} \psi\right\|_{X^{\prime}}
\end{aligned}
$$

and let $A=\max \left(A_{0}, A_{1}\right)$. Note, that $A_{0}=A_{1}$, if $k(x, y)=1$.

Theorem 1. Let $X$ and $Y$ be BFS satisfying the Berezhnoi $\ell$-condition and let $K$ be an integral operator of the form (1.1) with the kernel $k(x, y) \geq 0$ satisfying (1.2). Then $K: X \rightarrow Y$ is bounded, if and only if, $A<\infty$. Moreover,

$$
D^{-1} A \leq\|K\|_{X \rightarrow Y} \leq d_{1} d_{2} \gamma(D) A
$$

where $\gamma(D)$ depends only on $D$.

Remark 1. (i) The boundedness of $K$ was characterized in [14], [19] (for Lebesgue spaces) and in [11] (for Lorentz spaces.) The case $k(x, y)=1$ has been intensively studied for the last few decades by many authors and has led to further developments (sf. [15], [19]).

(ii) The Bereznoi $\ell$-condition corresponds to the case $p \leq q$ in the $L^{p}-L^{q}$ setting and to the case $\max (r, s) \leq \min (p, q)$ in the Lorentz $L^{r s}-L^{p q}$ setting, see $[\mathbf{6}],[\mathbf{1 1}]$. If no $\ell$-condition holds, then the lower bound in (3.3) is nevertheless valid. Moreover, there exists an operator, for which (3.3) is valid for spaces with no $\ell$-condition [17].

Theorem 2. Let the assumptions of Theorem 1 be fulfilled and suppose the spaces $X^{\prime}$ and $Y$ have $A C$-norms. Then $K: X \rightarrow Y$ is compact, if and only if $A<\infty$ and

$$
\lim _{t \rightarrow a_{i}} A_{i}(t)=\lim _{t \rightarrow b_{i}} A_{i}(t)=0 ; \quad i=0,1,
$$

where

(3.5) $a_{i}=\inf \left\{t>0: A_{i}(t)>0\right\}, b_{i}=\sup \left\{t>0: A_{i}(t)>0\right\} ; \quad i=0,1$.

Remark 2. In fact, it follows from the proof of Theorem 2 below, that $a_{0}=a_{1}, b_{0}=b_{1}$. 
The condition (3.4) has been formulated by many authors only for $a_{0}=a_{1}=0, b_{0}=b_{1}=\infty$. However, it is easy to find a formal counterexample, for which $A<\infty$ and (3.4) is valid with $a_{0}=a_{1}=0$, $b_{0}=b_{1}=\infty$, but $K$ is non-compact. The matter is, that the condition (3.4) has to be formulated for the end-points of the "real" interval of action of operator $K$ (see Remark 4 below for further details).

In the non-compact case we estimate the measure of non-compactness of the operator $K$ (or, equivalently, the distance of $K$ from the set of finite rank operators) defined by

$$
\alpha(K)=\inf \{\|K-P\| ; \operatorname{rank} P<\infty\} .
$$

To this end we need additional notation; put for all $0<a<z<b<\infty$ :

$$
\begin{aligned}
J_{L}^{0}(a) & =\sup _{0<t<a}\left\|\chi_{[t, a]} \varphi\right\|_{Y}\left\|\chi_{[0, t]}(\cdot) k(t, \cdot) \psi(\cdot)\right\|_{X^{\prime}}, \\
J_{L}^{1}(a) & =\sup _{0<t<a}\left\|\chi_{[t, a]}(\cdot) k(\cdot, t) \varphi(\cdot)\right\|_{Y}\left\|\chi_{[0, t]} \psi\right\|_{X^{\prime}}, \\
J_{L}(z) & =\max \left(J_{L}^{0}(z), J_{L}^{1}(z)\right), \quad J_{L}=\lim _{z \rightarrow a_{0}} J_{L}(z) ; \\
J_{R}^{0}(b) & =\sup _{b<t<\infty}\left\|\chi_{[t, \infty]} \varphi\right\|_{Y}\left\|\chi_{[b, t]}(\cdot) k(t, \cdot) \psi(\cdot)\right\|_{X^{\prime}}, \\
J_{R}^{1}(b) & =\sup _{b<t<\infty}\left\|\chi_{[t, \infty]}(\cdot) k(\cdot, t) \varphi(\cdot)\right\|_{Y}\left\|\chi_{[b, t]} \psi\right\|_{X^{\prime}} \\
J_{R}(z) & =\max \left(J_{R}^{0}(z), J_{R}^{1}(z)\right), \quad J_{R}=\lim _{z \rightarrow b_{0}} J_{R}(z) ; \\
J & =\max \left(J_{L}, J_{R}\right) .
\end{aligned}
$$

Theorem 3. Let the assumptions of Theorem 2 be valid and $K: X \rightarrow Y$ be bounded. Then

$$
D^{-1} J \leq \alpha(K) \leq d_{1}^{2} d_{2}^{2} \gamma(D) J
$$

Utilizing the scheme from $[\mathbf{1 1}]$ we estimate from above and below the approximation numbers of the Hardy operator of the form

$$
H f(x)=\varphi(x) \int_{0}^{x} \psi(y) f(y) d y
$$

This part of the paper has been initiated by D. E. Edmunds, W. D. Evans and D. J. Harris in the work [5]. Afterwards the extention for convolution operators with the polynomial kernel was given in [7] and for the Hardy operator in Lorentz spaces in [11]. The statement of the results and proofs of this part are given in Section 5 . 


\section{Boundedness, compactness} and measure of non-compactness

We begin with an alternative proof of the criterion for the boundedness of the Hardy operator due to E. Berezhnoi. Then we establish the proof for case in which the kernel satisfies Oinarov's condition. The basic idea is to apply the principle of duality to obtain the upper bound instead of using direct estimates.

Theorem 4 [3]. Let $X$ and $Y$ be BFS satisfying the $\ell$-condition, and let operator $H$ be defined by (3.8). Then $H: X \rightarrow Y$ is bounded if, and only if

$$
A=\sup _{t>0} A(t)=\sup _{t>0}\left\|\chi_{[t, \infty]} \varphi\right\|_{Y}\left\|\chi_{[0, t]} \psi\right\|_{X^{\prime}}<\infty
$$

Moreover,

$$
A \leq\|H\|_{X \rightarrow Y} \leq 4 d_{1} d_{2} A
$$

Proof: Necessity: For the lower bound we repeat the Berezhnoi argument [3]. If $H: X \rightarrow Y$ is bounded, then using axioms (2) and (3) of BFS we find for arbitrary $t>0$ and for all $f \in X$ such that $f(y) \psi(y) \geq 0$

$$
\begin{aligned}
\|H\|_{X \rightarrow Y}\|f\|_{X} & \geq\|H f\|_{Y}=\left\|\varphi(x) \int_{0}^{x} f(y) \psi(y) d y\right\|_{Y} \\
& \geq\left\|\chi_{[t, \infty)}(x) \varphi(x) \int_{0}^{x} f(y) \psi(y) d y\right\|_{Y} \\
& \geq\left\|\chi_{[t, \infty)}(x) \varphi(x)\right\|_{Y} \int_{0}^{t} f(y) \psi(y) d y \\
& =\left\|\chi_{[t, \infty)} \varphi\right\|_{Y} \int_{0}^{\infty} \chi_{[0, t]}(y) f(y) \psi(y) d y .
\end{aligned}
$$

Consequently, applying (2.1), we have $\|H\|_{X \rightarrow Y} \geq A(t)$ for all $t>0$ and it follows that $\|H\|_{X \rightarrow Y} \geq A$.

Sufficency: It follows from the principle of duality that for the upper bound it is sufficient to prove the estimate

$$
J \equiv\left|\int_{0}^{\infty} \varphi F g\right| \ll A\|f\|_{X}\|g\|_{Y^{\prime}}
$$


for all $f \in X$ and $g \in Y^{\prime}$, where

$$
F(x)=\int_{0}^{x} f(y) \psi(y) d y .
$$

Suppose, that $f(y) \psi(y) \neq 0$ on a set of positive measure, then we can choose a sequence $\left\{x_{k}\right\} \subset \mathbf{R}^{+}$such, that

$$
\int_{0}^{x_{k}}|f(y) \psi(y)| d y=2^{k}, \quad-\infty<k \leq N \leq \infty,
$$

where $N=\sup \left\{k: I_{k}=\left[x_{k-1}, x_{k}\right) \neq \emptyset\right\}$. Then, applying Hölder's inequality, (2.3), (2.5) and (4.1), we get

$$
\begin{aligned}
J & \leq \int_{0}^{\infty}|\varphi F g| \leq \sum_{k \leq N} 2^{k+1} \int_{I_{k+1}}|\varphi g| \\
& =4 \sum_{k \leq N} \int_{I_{k}}|f \psi| \int_{I_{k+1}}|\varphi g| \\
& \leq 4 \sum_{k \leq N}\left\|\chi_{I_{k}} f\right\|_{X}\left\|\chi_{I_{k}} \psi\right\|_{X^{\prime}}\left\|\chi_{I_{k+1}} \varphi\right\|_{Y}\left\|\chi_{I_{k+1}} g\right\|_{Y^{\prime}} \\
& \leq 4 A \sum_{k \leq N}\left\|\chi_{I_{k}} f\right\|_{X}\left\|\chi_{I_{k+1}} g\right\|_{Y^{\prime}} \\
& \leq 4 A\left\|\sum_{k} e_{k}\right\| \chi_{I_{k}} f\left\|_{X}\right\|_{\ell}\left\|\sum_{k} e_{k}\right\| \chi_{I_{k+1}} g\left\|_{Y^{\prime}}\right\|_{\ell^{\prime}} \\
& \leq 4 A d_{1} d_{2}\|f\|_{X}\|g\|_{Y^{\prime}} .
\end{aligned}
$$

Consequently, $\|H\|_{X \rightarrow Y} \leq 4 d_{1} d_{2} A$.

We shall need the following modification of Theorem 4.

Theorem 5. Let $X$ and $Y$ be BFS satisfying the $\ell$-condition and

$$
H_{\omega} f(x)=\varphi(x) \int_{0}^{\omega(x)} \psi(y) f(y) d y
$$

where $y=\omega(x)$ is a differentiable increasing function on $\mathbf{R}^{+}$such that $\omega(0)=0, \omega(\infty)=\infty$ and, thus, the inverse function $x=\omega^{-1}(y)$ exists. Then

$$
A_{\omega} \leq\left\|H_{\omega}\right\|_{X \rightarrow Y} \leq 4 d_{1} d_{2} A_{\omega}
$$

where

$$
A_{\omega}=\sup _{t>0}\left\|\chi_{[0, t]} \psi\right\|_{X^{\prime}}\left\|\chi_{\left[\omega^{-1}(t), \infty\right)} \varphi\right\|_{Y}=\sup _{t>0}\left\|\chi_{[0, \omega(t)]} \psi\right\|_{X^{\prime}}\left\|\chi_{[t, \infty)} \varphi\right\|_{Y} .
$$


Proof of Theorem 5: Is similar to the proof of Theorem 4. We omit details.

Proof of Theorem 1: Necessity: Note that the Oinarov condition (1.2) implies

$$
k(x, y) \geq D^{-1} k(t, y) \text { for all } x \geq t \geq y \geq 0 .
$$

Consequently, applying (4.3), we obtain for all $f \in X$ such that $f(y) \psi(y) \geq 0$

$$
\begin{array}{r}
\|K\|_{X \rightarrow Y}\|f\|_{X} \geq\left\|\varphi(x) \int_{0}^{x} k(x, y) f(y) \psi(y) d y\right\|_{Y} \\
\geq D^{-1}\left\|\chi_{[t, \infty)} \varphi\right\|_{Y} \int_{0}^{t} k(t, y) f(y) \psi(y) d y
\end{array}
$$

and arguing as in the necessity part of Theorem 4 we find, that $\|K\|_{X \rightarrow Y} \geq D^{-1} A_{0}$. By the principle of duality $\|K\|_{X \rightarrow Y}=\left\|K^{\prime}\right\|_{Y^{\prime} \rightarrow X^{\prime}}$, where

$$
K^{\prime} g(y)=\psi(y) \int_{y}^{\infty} k(x, y) \varphi(x) g(x) d x
$$

Applying the above argument to the operator $K^{\prime}$, we find $\left\|K^{\prime}\right\|_{X \rightarrow Y} \geq$ $D^{-1} A_{1}$ and, thus,

$$
\|K\|_{X \rightarrow Y} \geq D^{-1} A .
$$

For sufficiency we need the following two lemmas.

Lemma 2. Let $k_{0}(x, y) \geq 0, x \geq y \geq 0$ be nondecreasing and continuous with respect to $x$. Assume that

$$
k_{0}(x, y) \leq D_{0}\left(k_{0}(x, z)+k_{0}(z, y)\right), \quad x \geq z \geq y \geq 0
$$

with $D_{0} \geq 1$ independent of $x, z, y$. Let $f(y)$ be locally integrable, $\psi(y)$ be bounded and compactly supported and $f(y) \psi(y) \geq 0$. Let $G_{0}(x)=$ $\int_{0}^{x} k_{0}(x, y) \psi(y) f(y) d y$ be such, that $0<G_{0}(x)<\infty$ for some $x>0$. For a fixed number $\delta>0$ we define $\Delta_{k}=\left\{x>0: G_{0}(x) \geq(\delta+1)^{k}\right\}$, $k \in \mathbf{Z}, N=\max _{\Delta_{k} \neq \emptyset} k ; x_{k}=\inf \Delta_{k}, k \leq N, x_{N+1}=\infty$ if $N<\infty$. If $\delta \geq D_{0}$, then $0<\cdots<x_{k-1}<x_{k}<\cdots<x_{N}<\infty$ and the inequality

$$
\text { (4.4) } \begin{aligned}
& (\delta+1)^{k-1} \\
\leq & \int_{x_{k-1}}^{x_{k}} k_{0}\left(x_{k}, y\right) f(y) \psi(y) d y+D_{0} k_{0}\left(x_{k}, x_{k-1}\right) \int_{0}^{x_{k-1}} f(y) \psi(y) d y
\end{aligned}
$$

holds for all $k \leq N$. 
Proof of Lemma 2: Using the definition of $\left\{x_{k}\right\}$ and exploiting the property of $k_{0}(x, y)$, we find

$$
\begin{aligned}
(\delta+1)^{k-1}= & (\delta+1)^{k}-\delta(\delta+1)^{k-1} \leq G_{0}\left(x_{k}\right)-\delta(\delta+1)^{k-1} \\
= & \int_{0}^{x_{k}} k_{0}\left(x_{k}, y\right) f(y) \psi(y) d y-\delta(\delta+1)^{k-1} \\
= & \int_{0}^{x_{k-1}} k_{0}\left(x_{k}, y\right) f(y) \psi(y) d y \\
& +\int_{x_{k-1}}^{x_{k}} k_{0}\left(x_{k}, y\right) f(y) \psi(y) d y-\delta(\delta+1)^{k-1} \\
\leq & \int_{x_{k-1}}^{x_{k}} k_{0}\left(x_{k}, y\right) f(y) \psi(y) d y \\
& +D_{0} k_{0}\left(x_{k}, x_{k-1}\right) \int_{0}^{x_{k-1}} f(y) \psi(y) d y \\
& +D_{0} \int_{0}^{x_{k-1}} k_{0}\left(x_{k-1}, y\right) f(y) \psi(y) d y-\delta(\delta+1)^{k-1} .
\end{aligned}
$$

Now,

$$
\begin{aligned}
D_{0} \int_{0}^{x_{k-1}} k_{0}\left(x_{k-1}, y\right) f(y) \psi(y) d y & -\delta(\delta+1)^{k-1} \\
& =D_{0} G_{0}\left(x_{k-1}\right)-\delta(\delta+1)^{k-1} \leq 0
\end{aligned}
$$

provided $\delta \geq D_{0}$, and lemma is proved.

Remark 3. It follows from Lemma 2, that

$$
G_{0}(x) \leq(\delta+1)^{k}, \quad x \in\left[x_{k-1}, x_{k}\right), \quad k \leq N
$$

Lemma 3. Let $k(x, y) \geq 0$ satisfy (1.2) with $D \geq 1$ and let $k(x, y)$, $\varphi(x)$ and $\psi(y)$ be bounded functions such that $\operatorname{supp} \varphi=\operatorname{supp} \psi \subset(0, b)$, $b<\infty$. Then there exists $k_{h}(x, y) \geq 0,0<h<1$, satisfying Lemma 2 with $D_{h}=\max \left(2, D^{2}\right)$ such that $k(x, y) \leq k_{h}(x, y), 0<h<1$ and, moreover, if $A_{0, h}, A_{1, h}$ be defined by (3.1), (3.2) with $k_{h}(x, y)$ instead of $k(x, y)$, then

$$
A_{i} \leq \lim _{h \rightarrow+0} A_{i, h} \leq D A_{i}, \quad i=0,1
$$


Proof of Lemma 3: Put

$$
\bar{k}(x, y)=\sup _{y \leq t \leq x} k(t, y)
$$

Obviously, $\bar{k}(x, y)$ is nondecreasing with respect to $x$ and nonincreasing with respect to $y$ and $k(x, y) \leq \bar{k}(x, y)$. Moreover, (4.3) implies, that $\bar{k}(x, y) \leq D k(x, y)$. Hence,

$$
k(x, y) \leq \bar{k}(x, y) \leq D k(x, y)
$$

and

$$
\frac{1}{2}(\bar{k}(x, z)+\bar{k}(z, y)) \leq \bar{k}(x, y), \quad x \geq z \geq y \geq 0
$$

From the right hand side of (1.2) and (4.6) we have

$$
\begin{aligned}
\bar{k}(x, y) & \leq D^{2}(k(x, z)+k(z, y)) \\
& \leq D^{2}(\bar{k}(x, z)+\bar{k}(z, y)), \quad x \geq z \geq y \geq 0 .
\end{aligned}
$$

Consequently, $\bar{k}(x, y)$ satisfies Oinarov's condition with the constant $\bar{D}=\max \left(2, D^{2}\right)$. Define

$$
k_{h}(x, y)=\frac{1}{h} \int_{x}^{x+h} \bar{k}(t, y) d t, \quad 0<h<1 .
$$

Obviously, $k_{h}(x, y)$ is continuous and nondecreasing with respect to $x$ and nonincreasing in $y$ and

$$
k(x, y) \leq \bar{k}(x, y) \leq k_{h}(x, y)
$$

Hence, applying (4.8) we find

$$
\begin{aligned}
k_{h}(x, y) & \leq \frac{\bar{D}}{h} \int_{x}^{x+h}(\bar{k}(t, z)+\bar{k}(z, y)) d t=\bar{D}^{2}\left(k_{h}(x, z)+\bar{k}(z, y)\right) \\
& \leq \bar{D}^{2}\left(k_{h}(x, z)+k_{h}(z, y)\right), \quad x \geq z \geq y \geq 0
\end{aligned}
$$

Consequently, $k_{h}(x, y)$ satisfies Oinarov's condition with the constant $D_{h}=\bar{D}$. 
Let $A_{0, h}(t), A_{1, h}(t)$ be determined by (3.1) and (3.2) with $k_{h}$ instead of $k$, respectively. Then applying (4.6) and (4.10) we find

$A_{0}(t) \leq A_{0, h}(t)=\left\|\left(\chi_{[t+h, \infty)}-\chi_{[t+h, \infty)}+\chi_{[t, \infty)}\right) \varphi\right\|_{Y}\left\|\chi_{[0, t]} k_{h}(t, \cdot) \psi(\cdot)\right\|_{X^{\prime}}$

$$
\begin{aligned}
\leq & D\left\|\chi_{[t+h, \infty)} \varphi\right\|_{Y}\left\|\chi_{[0, t+h]}(\cdot) k(t+h, \cdot) \psi(\cdot)\right\|_{X^{\prime}} \\
& +\|\varphi\|_{\infty}\|k\|_{\infty}\|\psi\|_{\infty}\left\|\chi_{[0, b]}\right\|_{X^{\prime}}\left\|\chi_{[t, t+h]} \chi_{[0, b]}\right\|_{Y} .
\end{aligned}
$$

It implies

$$
A_{0} \leq A_{0, h} \leq D A_{0}+C \sup _{t>0}\left\|\chi_{[t, t+h]} \chi_{[0, b]}\right\|_{Y}
$$

with a finite constant $C$. Since $A_{0, h}$ decreases, when $h \rightarrow+0$, and $\left\|\chi_{[t, t+h]} \chi_{[0, b]}\right\|_{Y}$ is continuous in $t$ and compactly supported the result for $A_{0, h}$ follows from (4.13) by letting $h \rightarrow+0$. The argument for $A_{1, h}$ is analogous. Lemma 3 is proved.

Now we continue with the sufficient part of Theorem 1. By the principle of duality it is sufficient to show, that

$$
J \equiv\left|\int_{0}^{\infty} \varphi G g\right| \ll A\|f\|_{X}\|g\|_{Y^{\prime}}
$$

for all compactly supported $f \in X$ and $g \in Y^{\prime}$, where

$$
G(x)=\int_{0}^{x} k(x, y) f(y) \psi(y) d y
$$

Assume that $\varphi(x)$ and $\psi(y)$ are bounded compactly supported functions. Because of (4.10) we have

$$
J \leq \int_{0}^{\infty}|\varphi G g| \leq \int_{0}^{\infty}\left|\varphi G_{h} g\right|:=J_{h}
$$

where

$$
G_{h}(x)=\int_{0}^{x} k_{h}(x, y) f(y) \psi(y) d y
$$

and without loss of generality we take $f(y) \psi(y) \geq 0$. Hence, we may and shall apply Lemma 2 with $D_{0}=\max \left(2, D^{2}\right), \delta=D_{0}$ and the sequence 
of intervals $I_{k}=\left[x_{k-1}, x_{k}\right)$ with (4.4) holding. By Remark 2 we obtain

$$
\begin{aligned}
J_{h} \leq & \sum_{k \leq N} \int_{x_{k}}^{x_{k+1}}\left|\varphi G_{h} g\right| \leq \sum_{k \leq N}(\delta+1)^{k+1} \int_{x_{k}}^{x_{k+1}}|\varphi g| \\
\leq & (\delta+1)^{2} \sum_{k \leq N}(\delta+1)^{k-1} \int_{I_{k+1}}|\varphi g| \\
\leq & (\delta+1)^{2}\left[\sum_{k \leq N} \int_{I_{k}} k_{h}\left(x_{k}, y\right) \psi(y) f(y) d y \int_{I_{k+1}}|\varphi g|\right. \\
& \left.+D_{0} \sum_{k \leq N} k_{h}\left(x_{k}, x_{k-1}\right) \int_{0}^{x_{k-1}} \psi(y) f(y) d y \int_{I_{k+1}}|\varphi g|\right] \\
:= & (\delta+1)^{2}\left[J_{1, h}+D_{0} J_{2, h}\right] .
\end{aligned}
$$

Using the Hölder inequality, the $\ell$-condition with Lemma 1 and (4.13), we find

$$
\begin{aligned}
J_{1, h} & =\sum_{k \leq N} \int_{I_{k}} k_{h}\left(x_{k}, y\right) \psi(y) f(y) d y \int_{I_{k+1}}|\varphi g| \\
& \leq \sum_{k \leq N}\left\|\chi_{I_{k}} f\right\|_{X}\left\|\chi_{I_{k}}(\cdot) k_{h}\left(x_{k}, \cdot\right) \psi(\cdot)\right\|\left\|_{X^{\prime}}\right\| \chi_{I_{k+1}} \varphi\left\|_{Y}\right\| \chi_{I_{k+1}} g \|_{Y^{\prime}} \\
& \leq \sum_{k \leq N}\left\|\chi_{I_{k}} f\right\|_{X}\left\|\chi_{\left[0, x_{k}\right]}(\cdot) k_{h}\left(x_{k}, \cdot\right) \psi(\cdot)\right\|_{X^{\prime}}\left\|\chi_{\left[x_{k}, \infty\right)} \varphi\right\|_{Y}\left\|\chi_{I_{k+1}} g\right\|_{Y^{\prime}} \\
& \leq A_{0, h} \sum_{k \leq N_{0}}\left\|\chi_{I_{k}} f\right\|_{X}\left\|\chi_{I_{k+1}} g\right\|_{Y^{\prime}} \leq d_{1} d_{2} A_{0, h}\|f\|_{X}\|g\|_{Y^{\prime}} .
\end{aligned}
$$

For the term $J_{2, h}$ we write

$$
\begin{aligned}
J_{2, h}=\sum_{k \leq N} k_{h}\left(x_{k}, x_{k-1}\right)\left[\int_{0}^{x_{k-2}} \psi(y) f(y) d y\right. \\
\left.\quad+\int_{x_{k-2}}^{x_{k-1}} \psi(y) f(y) d y\right] \int_{I_{k+1}}|\varphi g|:=J_{2, h}^{(1)}+J_{2, h}^{(2)} .
\end{aligned}
$$

The estimate for $J_{2, h}^{(2)}$ is analogous to that for $J_{1, h}$. Applying the above scheme for estimating $J_{1, h}$ and using that $k_{h}(x, y)$ is non-increasing with 
respect to $y$, we get,

$$
\begin{aligned}
J_{2, h}^{(2)} & =\sum_{k \leq N} k_{h}\left(x_{k}, x_{k-1}\right) \int_{x_{k-2}}^{x_{k-1}} \psi(y) f(y) d y \int_{I_{k+1}}|\varphi g| \\
& \leq \sum_{k \leq N} \int_{I_{k-1}} k_{h}\left(x_{k}, y\right) \psi(y) f(y) d y \int_{I_{k+1}}|\varphi g| \\
(4.16) & \leq \sum_{k \leq N}\left\|\chi_{I_{k-1}} f\right\|_{X}\left\|\chi_{I_{k-1}}(\cdot) k_{h}\left(x_{k}, \cdot\right) \psi(\cdot)\right\|_{X^{\prime}}\left\|\chi_{I_{k+1}} \varphi\right\|_{Y}\left\|\chi_{I_{k+1}} g\right\|_{Y^{\prime}} \\
& \leq \sum_{k \leq N}\left\|\chi_{I_{k-1}} f\right\|_{X}\left\|\chi_{\left[0, x_{k}\right]}(\cdot) k_{h}\left(x_{k}, \cdot\right) \psi(\cdot)\right\|\left\|_{X^{\prime}}\right\| \chi_{\left[x_{k}, \infty\right)} \varphi\left\|_{Y}\right\|_{\chi_{I_{k+1}} g \|_{Y^{\prime}}} \\
& \leq d_{1} d_{2} A_{0, h}\|f\|_{X}\|g\|_{Y^{\prime}} .
\end{aligned}
$$

For the term $J_{2, h}^{(1)}$ we obtain

$$
\begin{aligned}
& J_{2, h}^{(1)}=\sum_{k \leq N} k_{h}\left(x_{k}, x_{k-1}\right) \int_{0}^{x_{k-2}} \psi(y) f(y) d y \int_{I_{k+1}}|\varphi g| \\
& =\int_{0}^{\infty}\left(\int_{0}^{\Omega(x)} f(y) \psi(y) d y\right) \sum_{k \leq N} k_{h}\left(x_{k}, x_{k-1}\right) \chi_{I_{k+1}}(x)|\varphi(x) g(x)| d x
\end{aligned}
$$

where

$$
\Omega(x)=\sum_{k \leq N} x_{k-2} \chi_{I_{k+1}}(x) .
$$

Let $y=\omega(x)$ be a function that satisfies the hypothesis of Theorem 5 and such that $\omega\left(x_{k}\right)=x_{k-2}, k \leq N ; \Omega(x) \leq \omega(x) \leq x, x>0$. Then

$$
J_{2, h}^{(1)} \leq \int_{0}^{\infty}\left(\int_{0}^{\omega(x)} f(y) \psi(y) d y\right) Q(x) g(x) d x,
$$

where

$$
Q(x)=\left(\sum_{k \leq N} k_{0}\left(x_{k}, x_{k-1}\right) \chi_{I_{k+1}}(x)\right) \varphi(x) .
$$

Applying Hölder's inequality and the upper bound in (4.2) of Theorem 5 we obtain

$$
\int_{0}^{\infty}\left(\int_{0}^{\omega(x)} f(y) \psi(y) d y\right) Q(x) g(x) d x \leq 4 d_{1} d_{2} \mathcal{A}_{\omega}\|f\|_{X}\|g\|_{Y^{\prime}}
$$


where

$$
\mathcal{A}_{\omega}=\sup _{t>0}\left\|\chi_{[0, t]} \psi\right\|_{X^{\prime}}\left\|\chi_{\left[\omega^{-1}(t), \infty\right)} Q\right\|_{Y}
$$

For a fixed number $t \in(0, \infty)$ there exists a union of disjoint intervals such that

$$
\left[\omega^{-1}(t), \infty\right) \subseteq \bigcup_{k_{0} \leq k \leq N} I_{k+1} .
$$

It is easy to see from the definition of the function $\omega(x)$, that $\omega^{-1}(t) \in$ $I_{k+1}$ if and only if $t \in I_{k-1}$ and for any $x \in\left[\omega^{-1}(t), \infty\right)$ we have two choices

$$
t<x_{k_{0}-1}<x_{k_{0}} \leq \omega^{-1}(t)<x<x_{k_{0}+1},
$$

when

$$
x \in\left[\omega^{-1}(t), \infty\right) \bigcap\left[x_{k_{0}}, x_{k_{0}+1}\right)
$$

or

$$
t<x_{k-1}<x_{k} \leq x<x_{k+1},
$$

when

$$
x \in I_{k+1}, \quad k>k_{0} .
$$

Since $k_{h}(x, y)$ is nondecreasing with respect to $x$ and nonincreasing with respect to $y$ we have in both cases

$$
k_{h}\left(x_{k}, x_{k-1}\right) \chi_{I_{k+1}}(x) \leq k_{h}(x, t), \quad k \geq k_{0} .
$$

Consequently,

$$
\left\|\chi_{\left[\omega^{-1}(t), \infty\right)} Q\right\|_{Y} \leq\left\|\chi_{[t, \infty)}(\cdot) k_{h}(\cdot, t) \varphi(\cdot)\right\|_{Y} .
$$

Using this and (4.17) we obtain that

$$
\mathcal{A}_{\omega} \leq A_{1, h}
$$

Thus,

$$
J_{2, h}^{(1)} \leq d_{1} d_{2} A_{1, h}\|f\|_{X}\|g\|_{Y^{\prime}} .
$$

Combining the estimates (4.12)-(4.18) and using Lebesgue's dominated convergence theorem we get the upper bound

$$
J \leq d_{1} d_{2} \gamma(D) A\|f\|_{X}\|g\|_{Y^{\prime}}
$$


where

$$
\gamma(D)=D\left(1+\max \left(2, D^{2}\right)\right)^{2}\left(1+2 \max \left(2, D^{2}\right)\right) .
$$

By Fatou's theorem we obtain (4.19) for $f, g, \varphi, \psi$ with no restriction. Theorem 1 is proved.

Remark 4. (i) There are three natural analogues of Theorem 1. The first is a restriction to an interval of real axis, the second deals with the associate operator and the third is concerned the non-Volterra case if the kernel is symmetric with respect to $x$ and $y$. We omit details.

(ii) Note, that if $k\left(x_{0}, y_{0}\right)=\infty$ for some $\infty>x_{0} \geq y_{0}>0$, then Oinarov's condition implies

$$
k(x, y)=\infty, \quad x \geq x_{0} \geq y_{0} \geq y>0 .
$$

Consequently, the convention $0 \cdot \infty=0$ yields, that $A<\infty$ is possible, only if

$$
\left\|\chi_{\left[x_{0}, \infty\right)} \varphi\right\|_{Y}+\left\|\chi_{\left[0, y_{0}\right]} \psi\right\|_{X^{\prime}}=0 .
$$

Thus, such an operator $K$ is actually reduced to the interval $\left[y_{0}, x_{0}\right]$, where it coincides with

$$
K_{0} f(x)=\varphi(x) \int_{y_{0}}^{x} k(x, y) \psi(y) f(y) d y, \quad y_{0} \leq x \leq x_{0},
$$

being the null-operator outside of the interval. Thus we may and shall assume the kernel to be bounded $k(x, y) \leq c_{\tau}<\infty$ on every domain of the form

$$
\Omega_{\tau}=\{(x, y): \infty>\tau \geq x \geq y \geq 0\}
$$

Proof of Theorem 2: Necessity: That $A=\max \left(A_{0}, A_{1}\right)<\infty$ follows from Theorem 1 . If $f \in X, f(y) \psi(y) \geq 0$ then exploiting Oinarov's condition, we find

$$
\begin{aligned}
\infty>A\|f\|_{X} \gg\|K f\|_{Y} & \geq\left\|\chi_{[t, \infty)} K f\right\|_{Y} \\
& \geq D^{-1}\left\|\chi_{[t, \infty)} \varphi\right\|_{Y} \int_{a_{0}}^{t} k(t, y) \psi(y) f(y) d y .
\end{aligned}
$$

Now, by the principle of duality for an arbitrary fixed $\gamma \in(0,1)$ we may find a function $f_{t}$, such that $\operatorname{supp} f_{t} \subseteq\left[a_{0}, t\right], f_{t}(y) \psi(y) \geq 0,\left\|f_{t}\right\|_{X}=1$ and

$$
\infty>A \gg\left\|K f_{t}\right\|_{Y} \geq \gamma D^{-1}\left\|\chi_{[t, \infty)} \varphi\right\|_{Y}\left\|\chi_{\left[a_{0}, t\right]} k(t, \cdot) \psi(\cdot)\right\|_{X^{\prime}} .
$$


Given $G \in X^{\prime}$ the Hölder inequality and absolute continuity of the norm in $X^{\prime}$ yield

$$
\left|\int_{0}^{\infty} f_{t} G\right| \leq\left\|\chi_{\left[a_{0}, t\right]} G\right\|_{X^{\prime}} \rightarrow 0, \quad t \rightarrow a_{0} .
$$

Since $K^{\prime}: Y^{\prime} \rightarrow X^{\prime}$ is also a compact operator, for any given $\varepsilon>0$ there exists a finite number of functions $G_{1}, G_{2}, \ldots, G_{n_{\varepsilon}}$ such that

$$
\min _{1 \leq n \leq n_{\varepsilon}}\left\|K^{\prime} g-G_{n}\right\|_{X^{\prime}} \leq \varepsilon
$$

for every $g \in Y^{\prime},\|g\|_{Y^{\prime}} \leq 1$. Given $\varepsilon>0$ and $f_{t}$ with $\left\|f_{t}\right\|_{X}=1$ we find by the principle of duality and (4.23) $g \in Y^{\prime},\|g\|_{Y^{\prime}} \leq 1$ and $G_{n}$, such that

$$
\left\|K f_{t}\right\|_{Y} \leq(1-\varepsilon)\left|\int_{0}^{\infty} K f_{t} g\right|
$$

and

$$
\left\|K^{\prime} g-G_{n}\right\|_{X^{\prime}} \leq \varepsilon
$$

respectively, and using (4.21) we obtain

$$
\begin{aligned}
\left\|K f_{t}\right\|_{Y} & \leq(1-\varepsilon)\left|\int_{0}^{\infty} f_{t} K^{\prime} g\right| \\
& \leq(1-\varepsilon) \varepsilon+(1-\varepsilon)\left|\int_{0}^{\infty} f_{t} G_{n}\right| \leq \varepsilon, \quad t \rightarrow a_{0} .
\end{aligned}
$$

Consequently, $\left\|K f_{t}\right\|_{Y} \rightarrow 0, t \rightarrow a_{0}$, and a part of (3.4), namely $\lim _{t \rightarrow a_{0}} A_{0}(t)=0$, now follows from (4.21). Analogously, begining with the inequality

$$
\begin{aligned}
\infty>A \gg\|K f\|_{Y} \geq & \left\|\chi_{[t, \infty)} K f\right\|_{Y} \\
& \geq D^{-1}\left\|\chi_{[t, \infty)}(\cdot) \varphi(\cdot) k(\cdot, t)\right\|_{Y} \int_{a_{1}}^{t} \psi(y) f(y) d y,
\end{aligned}
$$

we prove, that $\lim _{t \rightarrow a_{1}} A_{1}(t)=0$. The dual assertions on infinity follows from the similar observations for the associate operator. For proving sufficiency we need the following result.

Lemma 4. Let $X$ and $Y$ be BFS on a separable $\sigma$-finite measure space and $T: X \rightarrow Y$ be an integral operator of the form $T f(\alpha)=$ $\int T(\alpha, \beta) f(\beta) d \beta$. If both $X^{\prime}$ and $Y$ have $A C$-norms and

$$
A_{T}=\|\| T(\alpha, \cdot)\left\|_{X^{\prime}}\right\|_{Y}<\infty
$$

then $T$ is compact. 
Proof of Lemma 4: It is sufficient to establish, that the set of the functions of the form

$$
\sum_{i=1}^{i_{0}} \mu_{i}(\alpha) \eta_{i}(\beta)
$$

is dense in the space $Y\left[X^{\prime}\right]$ with the norm defined by the right side of (4.24), where $\mu_{i} \in Y$ and $\eta_{i} \in X^{\prime}$. On the strength of ([9, Chapter XI, Lemma 2]) it is true if the both spaces $X^{\prime}$ and $Y$ are "order continuous". This is fulfilled, when $X^{\prime}$ and $Y$ have AC-norms, because of ([1, Chapter 1, Proposition 3.5]), and so the Lemma is proved.

We continue the proof of the sufficiency part of Theorem 2. Let us show first, that

$$
a_{0}=a_{1}, \quad b_{0}=b_{1}
$$

To this end assume, for instance, that $0 \leq a_{0}<a_{1}$. Then $A_{0}(t)=$ $A_{1}(t)=0, t \in\left[0, a_{0}\right]$ and it follows from the Landau resonance theorem ([1, Lemma 2.6]) and Theorem 1 , restricted to the interval $\left[0, a_{0}\right]$, that for a.e. $x \in\left[0, a_{0}\right]$

$$
\varphi(x) k(x, y) \psi(y)=0 \text { for a.e. } y \in[0, x] .
$$

From (3.5) we find, that

$$
A_{1}(t)=\left\|\chi_{[t, \infty)}(\cdot) k(\cdot, t) \varphi(\cdot)\right\|_{Y}\left\|\chi_{[0, t]} \psi\right\|_{X^{\prime}}=0, \quad a_{0}<t \leq a_{1} .
$$

If

$$
\left\|\chi_{[0, t]} \psi\right\|_{X^{\prime}}=0, \quad a_{0}<t \leq a_{1},
$$

then $\psi(y)=0$ for a.e. $y \in[0, t]$ by the first axiom of BFS and, hence, $A_{0}(t)=0, t>a_{0}$, which contradicts the definition of $a_{0}$. If

$$
\left\|\chi_{[t, \infty)}(\cdot) k(\cdot, t) \varphi(\cdot)\right\|_{Y}=0, \quad a_{0}<t \leq a_{1},
$$

then for all $a_{0}<t \leq a_{1}$

$$
\varphi(x) k(x, t)=0 \text { for a.e. } x \in[t, \infty)
$$

and for all $g \in Y^{\prime}$ such, that supp $g \subseteq\left[a_{0}, a_{1}\right], \varphi(x) g(x) \geq 0$ and arbitrary $f \in X$ such, that $f(t) \psi(t) \geq 0$, we find

$$
\begin{aligned}
\int_{a_{0}}^{a_{1}} K f(x) g(x) d x & =\int_{a_{0}}^{a_{1}} \varphi(x) g(x) d x \int_{a_{0}}^{x} k(x, t) \psi(t) f(t) d t \\
& =\int_{a_{0}}^{a_{1}} \psi(t) f(t) d t \int_{t}^{a_{1}} \varphi(x) k(x, t) g(x) d x=0
\end{aligned}
$$


and, again by the Landau theorem, we get for a.e. $x \in\left[a_{0}, a_{1}\right]$

$$
\varphi(x) k(x, y) \psi(y)=0 \text { for a.e. } y \in\left[a_{0}, x\right] .
$$

Now by (4.26) and (4.27)

$$
\int_{0}^{a_{1}} K f(x) g(x) d x=\int_{a_{0}}^{a_{1}} \varphi(x) g(x) d x \int_{0}^{a_{0}} k(x, t) \psi(t) f(t) d t
$$

and by the Hölder inequality and Oinarov's condition we find

$$
\begin{aligned}
& \left|\int_{0}^{a_{1}} K f(x) g(x) d x\right| \\
& \leq D \int_{a_{0}}^{a_{1}} k\left(x, a_{0}\right)|\varphi(x) g(x)| d x \int_{0}^{a_{0}}|\psi(t) f(t)| d t \\
& \quad+D \int_{a_{0}}^{a_{1}}|\varphi(x) g(x)| d x \int_{0}^{a_{0}} k\left(a_{0}, t\right)|\psi(t) f(t)| d t \\
& \leq D\left(\left\|\chi_{\left[a_{0}, a_{1}\right]}(\cdot) \varphi(\cdot) k\left(\cdot, a_{0}\right)\right\|_{Y}\left\|\chi_{\left[0, a_{0}\right]} \psi\right\|_{X^{\prime}}\right. \\
& \left.\quad+\left\|\chi_{\left[a_{0}, a_{1}\right]} \varphi\right\|_{Y}\left\|\chi_{\left[0, a_{0}\right]}(\cdot) k\left(a_{0}, \cdot\right) \psi(\cdot)\right\|_{X^{\prime}}\right)\left\|\chi_{\left[0, a_{1}\right]} g\right\|_{Y^{\prime}}\left\|\chi_{\left[0, a_{1}\right]} f\right\|_{X} \\
& \leq D\left(A_{0}\left(a_{0}\right)+A_{1}\left(a_{0}\right)\right)\left\|\chi_{\left[0, a_{1}\right]} g\right\|_{Y^{\prime}}\left\|\chi_{\left[0, a_{1}\right]} f\right\|_{X}=0 .
\end{aligned}
$$

Hence, by the principle of duality we obtain $\|K\|_{X_{\left[0, a_{1}\right]} \rightarrow Y_{\left[0, a_{1}\right]}}=0$, and, in particular, Theorem 1 , restricted to the interval $\left[0, a_{1}\right]$, implies $A_{0}(t)=0,0 \leq t \leq a_{1}$. Thus, $a_{0}=a_{1}$, and by similar arguments it can be proved that $b_{0}=b_{1}$. For this reason we may and shall assume further for simplicity, that $a_{0}=a_{1}=0, b_{0}=b_{1}=\infty$.

Let $0<a<b<\infty$ and put

$$
P_{a} f=\chi_{[0, a]} f, \quad Q_{b} f=\chi_{[b, \infty)} f, \quad P_{a b} f=\chi_{[a, b]} f .
$$

Then we have

(4.28) $K f=\left(P_{a}+P_{a b}+Q_{b}\right) K\left(P_{a}+P_{a b}+Q_{b}\right) f$

$$
=P_{a} K P_{a} f+Q_{b} K Q_{b} f+P_{a b} K P_{a b} f+Q_{b} K P_{a} f+Q_{b} K P_{a b} f+P_{a b} K P_{a} f .
$$

By Theorem 1 restricted to the intervals $[0, a]$ or $[b, \infty)$ and $(3.4)$ we have

$$
\begin{aligned}
& \left\|P_{a} K P_{a}\right\| \leq\left\{\sup _{0<t<a} A_{0}(t)+\sup _{0<t<a} A_{1}(t)\right\} \rightarrow 0, \quad a \rightarrow 0 \\
& \left\|Q_{b} K Q_{b}\right\| \leq\left\{\sup _{t>b} A_{0}(t)+\sup _{t>b} A_{1}(t)\right\} \rightarrow 0, \quad b \rightarrow \infty .
\end{aligned}
$$


It follows from Lemma 4 that the operator $Q_{b} K P_{a}$ is compact. Indeed,

$$
\begin{aligned}
A_{Q_{b} K P_{a}} \leq & \|\| \chi_{[0, b]}(\cdot) k(x, \cdot) \psi(\cdot)\left\|_{X^{\prime}} \chi_{[b, \infty)}(x) \varphi(x)\right\|_{Y} \\
\leq & D\left(\left\|\chi_{[b, \infty)}(x) k(x, b) \varphi(x)\right\|_{Y}\left\|\chi_{[0, b]} \psi\right\|_{X^{\prime}}\right. \\
& \left.+\left\|\chi_{[b, \infty)} \varphi\right\|_{Y}\left\|\chi_{[0, b]}(\cdot) k(b, \cdot) \psi(\cdot)\right\|_{X^{\prime}}\right) \\
\leq & D\left(A_{0}(b)+A_{1}(b)\right)<\infty
\end{aligned}
$$

Anagously, we find

$$
\begin{aligned}
& A_{Q_{b} K P_{a b}} \leq D\left(A_{0}(b)+A_{1}(b)\right)<\infty \\
& A_{P_{a b} K P_{a}} \leq D\left(A_{0}(a)+A_{1}(a)\right)<\infty .
\end{aligned}
$$

Note, that $0<\left\|\chi_{[a, \infty)} \varphi\right\|_{Y},\left\|\chi_{[0, b]} \psi\right\|_{X^{\prime}}<\infty$, otherwise $A_{0}(a)=A_{1}(b)=0$, and

$$
k(x, y) \leq c_{b}<\infty, \quad b \geq x \geq y \geq a .
$$

By Remark 4(ii), we may write

$$
\begin{aligned}
A_{P_{a b} K P_{a b}}=\|\| \chi_{[a, b]}(\cdot) k(x, \cdot) \psi(\cdot) \|_{X^{\prime}} \chi_{[a, b]}(x) \varphi(x) & \|_{Y} \\
& \leq c_{b}\left\|\chi_{[a, \infty)} \varphi\right\|_{Y}\left\|\chi_{[0, b]} \psi\right\|_{X^{\prime}}<\infty
\end{aligned}
$$

and hence, by Lemma 4 , operator $P_{a b} K P_{a b}$ is compact too. Using this and (4.29)-(4.31) we see, that $K$ is a limit of compact operators. This ends the proof of Theorem 2 .

Proof of Theorem 3: We assume for simplicity, that $a_{0}=a_{1}=0$, $b_{0}=b_{1}=\infty$. Let $0<a<b<\infty$. By Theorem 1 we obtain

$$
\begin{aligned}
& D^{-1} J_{L}(a) \leq\left\|P_{a} K P_{a}\right\| \leq d_{1} d_{2} \gamma(D) J_{L}(a), \\
& D^{-1} J_{R}(b) \leq\left\|Q_{b} K Q_{b}\right\| \leq d_{1} d_{2} \gamma(D) J_{R}(b),
\end{aligned}
$$

where the constant $\gamma(D)$ is defined by (4.20). Now, using (4.28) and taking into account the compactness of the last four components there, we see, that,

$$
\alpha(K) \leq\left\|P_{a} K P_{a}+Q_{b} K Q_{b}\right\| .
$$

Put $S=P_{a} K P_{a}$ and $T=Q_{b} K Q_{b}$. Then

$$
\begin{aligned}
\|S+T\| & =\sup _{f \neq 0} \frac{\|S f+T f\|_{Y}}{\|f\|_{X}} \\
& \leq d_{2} \sup \left\{\frac{\|\| S f\left\|_{Y}+\right\| T f\left\|_{Y}\right\|_{\ell}}{\|f\|_{X}} ; f \neq 0, f \psi \geq 0, P_{a b} f=0\right\} .
\end{aligned}
$$


If $P_{a b} f=0$, then by the Berezhnoi $\ell$-condition

$$
\|f\|_{X} \geq d_{1}^{-1}\|\| g\left\|_{X}+\right\| h\left\|_{X}\right\|_{\ell}
$$

where $g=P_{a} f$ and $h=Q_{b} f$. Hence,

$$
\begin{aligned}
\|S+T\| \leq & d_{1} d_{2} \sup _{f \neq 0, f=g+h} \frac{\|S f\|_{Y}+\|T f\|_{Y} \|_{\ell}}{\|\| g\left\|_{X}+\right\| h\left\|_{X}\right\|_{\ell}} \\
= & d_{1} d_{2} \sup _{f \neq 0, f=g+h} \| \frac{\|S g\|_{Y}}{\|g\|_{X}} \cdot \frac{\|g\|_{X}}{\|\| g\left\|_{X}+\right\| h\left\|_{X}\right\|_{\ell}} \\
& +\frac{\|T h\|_{Y}}{\|h\|_{X}} \cdot \frac{\|h\|_{X}}{\|\| g\left\|_{X}+\right\| h\left\|_{X}\right\|_{\ell} \|_{\ell}} \\
\leq & d_{1}^{2} d_{2}^{2} \gamma(D)\left\|J_{L}(a) \frac{\|g\|_{X}}{\|\| g\left\|_{X}+\right\| h\left\|_{X}\right\|_{\ell}}+J_{R}(b) \frac{\|h\|_{X}}{\|n\|_{X}+\|h\|_{X} \|_{\ell}}\right\|_{\ell} \\
\leq & d_{1}^{2} d_{2}^{2} \gamma(D) J .
\end{aligned}
$$

To obtain the lower bound, let $\theta>\alpha(K)$. If $Y$ has AC-norm, then $Y$ is separable [1]. Hence, there exists $T: X \rightarrow Y$ such that $\operatorname{rank} T<\infty$ and $\|K f-T f\|_{Y} \leq \theta\|f\|_{X}$ for all $f \in X$. Since range of the operator $T$ is formed by a finite number of functions from $Y$, we can approximate each of them by a bounded function with compact support [1] and, thus, given $\varepsilon>0$, there exist $T_{0}: X \rightarrow Y$ with $\operatorname{rank} T_{0}=\operatorname{rank} T$ and the numbers $0<\delta<N<\infty$, such that

$$
\left\|T-T_{0}\right\|<\varepsilon \operatorname{supp} T_{0} f \subset[\delta, N] \text { for all } f \in X .
$$

Hence,

$$
\left\|K f-T_{0} f\right\|_{Y} \leq(\theta+\varepsilon)\|f\|_{X} \text { for all } f \in X .
$$

Let $f$ be such that supp $f \subset[0, \delta] \bigcup[N, \infty)$ and $f \psi \geq 0$. Then

$(\theta+\varepsilon)\|f\|_{X} \geq\|K f\|_{Y}=\left\|K P_{\delta} f+K Q_{N} f\right\|_{Y} \geq\left\|P_{\delta} K P_{\delta} f+Q_{N} K Q_{N} f\right\|_{Y}$, since all the functions involved are non-negative. Thus

$$
(\theta+\varepsilon)\|f\|_{X} \geq\left\|P_{\delta} K P_{\delta} f\right\|_{Y}
$$


for all $f \in X$ with supp $f \subset[0, \delta]$ and $f \psi \geq 0$ and

$$
(\theta+\varepsilon)\|f\|_{X} \geq\left\|Q_{N} K Q_{N} f\right\|_{Y}
$$

for all $f \in X$ with $\operatorname{supp} f \subset[N, \infty)$ and $f \psi \geq 0$. Hence, applying the lower bound from Theorem 1, we obtain

$$
(\theta+\varepsilon) \geq D^{-1} J_{L}(\delta) \text { and }(\theta+\varepsilon) \geq D^{-1} J_{R}(N),
$$

Letting $\theta \rightarrow \alpha(K), \varepsilon \rightarrow 0$ and then $\delta \rightarrow 0, N \rightarrow \infty$ we establish the lower bound. Theorem 3 is proved.

Remark 5. Theorem 3 for Lebesgue spaces was proved in [7], the case $k(x, y)=1$ was given in $[\mathbf{6}]$.

\section{Approximation numbers}

We begin with the reminder, that for any positive integer $m$, the $m$-th approximation number $a_{m}$ of a bounded linear map $T: X \rightarrow Y$ is defined by

$$
a_{m}(T)=\inf \{\|T-P\| ; P \text { a bounded linear operator }
$$

$$
\text { and } \operatorname{rank} P<m\} \text {. }
$$

For further information on the approximation numbers we refer the reader to the monographs $[\mathbf{4}],[\mathbf{8}]$ and $[\mathbf{1 6}]$. We consider the operator $H: X \rightarrow Y$ of the form (3.8) and suppose, that $H$ is compact. We also assume for simplicity, that $a_{0}=0, b_{0}=\infty$ for the operator $H$. By Theorem 2 we get

$$
\begin{gathered}
A=\sup _{t>0} A(t)=\sup _{t>0}\left\|\chi_{[t, \infty]} \varphi\right\|_{Y}\left\|\chi_{[0, t]} \psi\right\|_{X^{\prime}}<\infty, \\
\lim _{t \rightarrow 0} A(t)=\lim _{t \rightarrow \infty} A(t)=0 .
\end{gathered}
$$

Given sufficiently small $\varepsilon, 0<\varepsilon<\|H\|$, we choose the numbers $0=c_{0}<$ $c_{1}<c_{2}<\cdots<c_{N-1}<c_{N}<c_{N+1}=\infty$ and intervals $I_{k}=\left[c_{k}, c_{k+1}\right]$, $k=0,1, \ldots, N$, such that

$$
A\left[c_{1}\right]=A\left[c_{N}\right]=\varepsilon,
$$

where

$$
\begin{aligned}
& A\left[c_{1}\right]=\sup _{0<t<c_{1}} A(t)=\sup _{0<t<c_{1}}\left\|\chi_{\left[t, c_{1}\right]} \varphi\right\|_{Y}\left\|\chi_{[0, t]} \psi\right\|_{X^{\prime}} \\
& A\left[c_{N}\right]=\sup _{c_{N}<t<\infty} A(t)=\sup _{c_{N}<t<\infty}\left\|\chi_{[t, \infty]} \varphi\right\|_{Y}\left\|\chi_{\left[c_{N}, t\right]} \psi\right\|_{X^{\prime}} .
\end{aligned}
$$


Lemma 5. Let $X$ and $Y$ be BFS satisfying the Berezhnoi $\ell$-condition, and suppose $Y$ and $Y^{\prime}$ have $A C$-norms. Let $0<a<b<\infty, I=(a, b)$ and

$$
\begin{aligned}
F(x) & =\int_{a}^{x} \psi(y) f(y) d y, \quad a \leq x \leq b ; \\
F_{I} & =\frac{1}{\mu(I)} \int_{I} F d \mu, \\
\mu(I) & =\int_{I} d \mu
\end{aligned}
$$

where $d \mu(x)=\varphi(x) g(x) d x$, and $g(x)$ is a function on I satisfying the inequality

$$
(1-\delta)\left\|\chi_{[a, b]} \varphi\right\|_{Y}\left\|\chi_{[a, b]} g\right\|_{Y^{\prime}} \leq \int_{I} \varphi(x) g(x) d x
$$

for a sufficiently small $0<\delta \leq 0,01$. Then

$$
\frac{3}{10} \max \left(B_{0}, B_{1}\right) \leq \sup _{f \neq 0} \frac{\left\|\chi_{[a, b]} \varphi\left(F-F_{I}\right)\right\|_{Y}}{\left\|\chi_{[a, b]} f\right\|_{X}} \leq 8 \frac{2}{25} d_{1}^{2} d_{2}^{2} \max \left(B_{0}, B_{1}\right),
$$

where

$$
\begin{aligned}
& B_{0}=\sup _{a<x<c}\left\|\chi_{[x, c]} \psi\right\|_{X^{\prime}}\left\|\chi_{[a, x]} \varphi\right\|_{Y}, \\
& B_{1}=\sup _{c<x<b}\left\|\chi_{[c, x]} \psi\right\|_{X^{\prime}}\left\|\chi_{[x, b]} \varphi\right\|_{Y} .
\end{aligned}
$$

Proof of Lemma 5: Given $f \in X_{[a, b]}, c \in(a, b)$ we put

$$
\Psi_{c}(x)= \begin{cases}-\int_{x}^{c} \psi(y) f(y) d y, & a \leq x<c, \\ \int_{c}^{x} \psi(y) f(y) d y, & c \leq x<b\end{cases}
$$

and $\Psi_{c, I}=\frac{1}{\mu(I)} \int_{I} \Psi_{c} d \mu$. Then

$$
F(x)-F_{I}=\Psi_{c}(x)-\Psi_{c, I} .
$$

To obtain the lower bound, we take $f \in X_{[a, b]}$ such that supp $f \subseteq[a, c]$ and suppose, that the inequality

$$
\left\|\chi_{[a, b]} \varphi\left(F-F_{I}\right)\right\|_{Y} \leq C\left\|\chi_{[a, b]} f\right\|_{X}
$$


holds for all $f \in X_{[a, b]}$ with a constant $C$ independent of $f$. Then

$$
\begin{aligned}
C\left\|\chi_{[a, c]} f\right\|_{X} & \geq\left\|\chi_{[a, c]} \varphi\left(F-F_{I}\right)\right\|_{Y}=\left\|\chi_{[a, c]} \varphi\left(\Psi_{c}-\Psi_{c, I}\right)\right\|_{Y} \\
& \geq\left\|\chi_{[a, c]} \varphi \Psi_{c}\right\|_{Y}-\left|\Psi_{c, I}\right|\left\|\chi_{[a, c]} \varphi\right\|_{Y} \\
& =\left\|\chi_{[a, c]} \varphi \Psi_{c}\right\|_{Y}-\frac{1}{\mu(I)}\left|\int_{I} \Psi_{c} d \mu\right|\left\|\chi_{[a, c]} \varphi\right\|_{Y} \\
& \geq\left\|\chi_{[a, c]} \varphi \Psi_{c}\right\|_{Y}-\frac{1}{\mu(I)} \int_{I}\left|\Psi_{c}\right| d \mu\left\|\chi_{[a, c]} \varphi\right\|_{Y} \\
& =\left\|\chi_{[a, c]} \varphi \Psi_{c}\right\|_{Y}-\frac{1}{\mu(I)} \int_{I}\left|\Psi_{c} \varphi \varphi^{-1}\right| d \mu\left\|\chi_{[a, c]} \varphi\right\|_{Y} \\
& \geq\left\|\chi_{[a, c]} \varphi \Psi_{c}\right\|_{Y}-\frac{1}{\mu(I)}\left\|\chi_{[a, c]} \varphi \Psi_{c}\right\|_{Y}\left\|\chi_{[a, c]} g\right\|_{Y^{\prime}} \|_{\chi_{[a, c]} \varphi \|_{Y}} \\
& =\left(1-\frac{1}{\mu(I)}\left\|\chi_{[a, c]} g\right\|_{Y^{\prime}}\left\|\chi_{[a, c]} \varphi\right\|_{Y}\right)\left\|\chi_{[a, c]} \varphi \Psi_{c}\right\|_{Y} \\
& =\left(1-\frac{V(a, c)}{\mu(I)}\right)\left\|\chi_{[a, c]} \varphi \Psi_{c}\right\|_{Y},
\end{aligned}
$$

where $V(a, c)=\left\|\chi_{[a, c]} \varphi\right\|_{Y}\left\|\chi_{[a, c]} g\right\|_{Y^{\prime}}$. Because of the absolute continuity of the norms $Y$ and $Y^{\prime}$, we can for any fixed $\beta \in(0,1-\delta)$ find a point $c \in(a, b)$ such that $V(a, c)=\beta \mu(I)$, therefore by Theorem 4 restricted to the interval $[a, c]$ we have $C \geq(1-\beta) B_{0}$. A similar argument applied for all $f$ such that $\operatorname{supp} f \subset[c, \bar{b})$ gives

$$
C\left\|\chi_{[c, b]} f\right\|_{X} \geq\left(1-\frac{W(c, b)}{\mu(I)}\right)\left\|\chi_{[c, b]} \varphi \Psi_{c}\right\|_{Y}
$$

where $W(c, b)=\left\|\chi_{[c, b]} \varphi\right\|_{Y}\left\|\chi_{[c, b]} g\right\|_{Y^{\prime}}$.

$$
\begin{aligned}
1-\frac{W(c, b)}{\mu(I)} & =\frac{1}{\mu(I)}(\mu(I)-W(c, b)) \\
& =\frac{1}{\mu(I)}\left((1-\delta)\left\|\chi_{[a, b]} \varphi\right\|_{Y}\left\|\chi_{[a, b]} g\right\|_{Y^{\prime}}-W(c, b)\right) \\
& \geq \beta(1-\delta)-\frac{W(c, b)}{\mu(I)} .
\end{aligned}
$$


If $c \rightarrow b$, then $\beta \rightarrow(1-\delta)$ and $W(c, b) \rightarrow 0$, therefore we can choose $c \in(a, b)$ such that

$$
\frac{W(c, b)}{\mu(I)} \leq \frac{\beta(1-\delta)}{2}
$$

By Theorem 4 we get $C \geq \frac{\beta(1-\delta)}{2} B_{1}$. Now, if we take $\beta$ such that $1-\beta=\frac{\beta(1-\delta)}{2}$, then $\beta=\frac{2}{3-\delta}$ and the required lower bound $C \geq$ $\frac{3}{10} \max \left(B_{0}, B_{1}\right)$.

Sufficiency: Using Hölder's inequality and the Berezhnoi $\ell$-condition, we see that

$$
\begin{aligned}
\left\|\chi_{[a, b]} \varphi\left(F-F_{I}\right)\right\|_{Y}= & \left\|\chi_{[a, b]} \varphi\left(\Psi_{c}-\Psi_{c, I}\right)\right\|_{Y} \\
\leq & \left\|\chi_{[a, b]} \varphi \Psi_{c}\right\|_{Y}+\left|\Psi_{c, I}\right|\left\|\chi_{[a, b]} \varphi\right\|_{Y} \\
\leq & \left\|\chi_{[a, b]} \varphi \Psi_{c}\right\|_{Y} \\
& +\frac{1}{\mu(I)}\left\|\chi_{[a, b]} \varphi \Psi_{c}\right\|_{Y}\left\|\chi_{[a, b]} g\right\|_{Y^{\prime}}\left\|\chi_{[a, b]} \varphi\right\|_{Y} \\
\leq & \frac{2}{(1-\delta)}\left\|\chi_{[a, b]} \varphi \Psi_{c}\right\|_{Y} \\
\leq & \frac{2}{(1-\delta)} d_{2}\|\| \chi_{[a, c]} \varphi \Psi_{c}\left\|_{Y}+\right\| \chi_{[c, b]} \varphi \Psi_{c}\left\|_{Y}\right\|_{\ell} \\
\leq & \frac{8 d_{1}^{2} d_{2}^{2}}{1-\delta} \max \left(B_{0}, B_{1}\right)\left\|\chi_{[a, b]} f\right\|_{X}
\end{aligned}
$$

and the required result follows. The proof of Lemma 5 is complete.

By Lemma 5 the norm of the operator

$$
H_{I} f(x)=\chi_{I}(x) \varphi(x)\left(F(x)-F_{I}\right)
$$

depends continuously on the interval $I$. We choose the intervals $I_{k}=$ $\left[c_{k}, c_{k+1}\right], k=1, \ldots, N-1$ so, that

$$
\begin{aligned}
\left\|H_{I_{k}}\right\| & =\varepsilon, \quad k=1, \ldots, N-2, \\
\left\|H_{N-1}\right\| & \leq \varepsilon .
\end{aligned}
$$


Now we follow the construction from ([7, Section 3.1]) adjusted to the present case. Let for $k=1, \ldots, N-1$

$$
\begin{aligned}
F_{k}(x) & =\chi_{I_{k}}(x) \int_{c_{k}}^{x} \psi(y) f(y) d y \\
P_{k} f(x) & =\chi_{I_{k}}(x)\left\{H f(x)-\varphi(x)\left(F_{k}(x)-F_{k, I_{k}}\right)\right\} .
\end{aligned}
$$

Observe, that the operator $P=\sum_{k=1}^{N-1} P_{k}$ is a bounded linear operator $P: X \rightarrow Y$ and $\operatorname{rank} P \leq N-1$.

Theorem 6. Let $X$ and $Y$ be BFS satisfying the Berezhnoi $\ell$-condition, and $X^{\prime}, Y, Y^{\prime}$ have $A C$ - norms. Let $H: X \rightarrow Y$ defined by (3.8) be a compact operator. Given $\varepsilon>0,\|H\|>\varepsilon$, let the integer $N>2$ and intervals $I_{k}=\left[c_{k}, c_{k+1}\right], k=0,1, \ldots, N$ be chosen so that (5.3) and (5.6) hold. Then the upper bound

$$
a_{N}(H) \leq d_{1} d_{2} \varepsilon
$$

is valid, where the constants $d_{1}, d_{2}$ are determined by (2.3), (2.4).

Proof of Theorem 6: Using Theorem 4 and the Berezhnoi $\ell$-condition we see that

$$
\begin{array}{r}
\|H f-P f\|_{Y} \leq d_{2}\|\varepsilon\| \chi_{\left[0, c_{1}\right]} f\left\|_{X}+\varepsilon\right\| \chi_{\left[c_{N}, \infty\right)} f\left\|_{X}+\sum_{k=1}^{N-1}\right\| H_{I_{k}} f\left\|_{Y}\right\|_{\ell} \\
\leq \varepsilon d_{2}\left\|\sum_{k=0}^{N}\right\| \chi_{I_{k}} f\left\|_{X}\right\|_{\ell} \leq \varepsilon d_{1} d_{2}\|f\|_{X},
\end{array}
$$

and thus, $a_{N}(H) \leq d_{1} d_{2} \varepsilon$.

Theorem 7. Let the assumptions of Theorem 6 hold and, moreover, let $X$ be $\ell_{p_{1}}$-convex BFS and $Y$ be $\ell_{p_{2}}$-concave BFS for BSS $\ell_{p_{1}}, \ell_{p_{2}}$ with $p_{2} \geq p_{1}>1$. Then the following lower bound

$$
a_{N}(H) \geq \frac{1}{2,02} \varepsilon d_{1}^{-1} d_{2}^{-1} N^{\frac{1}{p_{2}}-\frac{1}{p_{1}}}
$$

holds. 
Proof of Theorem 7: Let $\lambda \in(0,1)$. We take the sequence of functions $f_{k} \in X$, such that $\operatorname{supp} f_{k} \subset I_{k}$, and

$$
\begin{gathered}
\frac{\left\|\chi_{I_{i}} \varphi F_{i}\right\|_{Y}}{\left\|f_{i}\right\|_{X}} \geq \lambda \varepsilon, \quad i=0, N \\
\frac{\left\|\chi_{I_{k}} \varphi\left(F_{k}-F_{k, I_{k}}\right)\right\|_{Y}}{\left\|f_{k}\right\|_{X}} \geq \lambda \varepsilon, \quad k=1,2, \ldots, N-1,
\end{gathered}
$$

where

$$
F_{k}(x)=\int_{c_{k}}^{x} \psi(y) f_{k}(y) d y, \quad k=0,1, \ldots, N-1 .
$$

In this construction we follow [5]. Let $\widetilde{P}: X \rightarrow Y$ be a bounded linear map and $\operatorname{rank} \widetilde{P} \leq N$. Then we choose constants $\nu_{0}, \nu_{1}, \nu_{2}, \ldots, \nu_{N}$ such that

$$
\widetilde{P}\left(\sum_{k=0}^{N} \nu_{k} f_{k}\right)=0
$$

Put $f=\sum_{k=0}^{N} \nu_{k} f_{k}$ and as before

$$
F(x)=\int_{0}^{x} \psi(y) f(y) d y, \quad x>0
$$

For all $x \in I_{k}$

$$
F(x)=\nu_{k} F_{k}(x)+\mu_{k}, \quad k=0,1, \ldots, N-1,
$$

for some constant $\mu_{k}$. For all $c \in \mathbf{R}$

$$
\begin{aligned}
\left\|\chi_{I} \varphi\left(F-F_{I}\right)\right\|_{Y} & \leq\left\|\chi_{I} \varphi\left(F-c-(F-c)_{I}\right)\right\|_{Y} \\
& \leq\left\|\chi_{I} \varphi(F-c)\right\|_{Y}+\left|(F-c)_{I}\right|\left\|\chi_{I} \varphi\right\|_{Y} \\
& \leq \frac{2}{1-\delta}\left\|\chi_{I} \varphi(F-c)\right\|_{Y} \leq 2,02\left\|\chi_{I} \varphi(F-c)\right\|_{Y} .
\end{aligned}
$$

Hence,

$$
\left\|\chi_{I} \varphi\left(F-F_{I}\right)\right\|_{Y} \leq 2,02 \inf _{c \in \mathbf{R}}\left\|\chi_{I} \varphi(F-c)\right\|_{Y} .
$$


Applying (5.9)-(5.12), $\ell_{p_{2}}$-concavity of $Y$ and $\ell_{p_{1}}$-convexity of $X$, we find

$$
\begin{aligned}
& \|H f-\widetilde{P} f\|_{Y}^{p_{2}}=\|H f\|_{Y}^{p_{2}} \\
& \geq d_{1}^{-p_{2}}\left(\left\|\chi_{I_{0}} \varphi F_{0}\right\|_{Y}^{p_{2}}+\sum_{k=1}^{N-1}\left\|\chi_{I_{k}} \varphi F\right\|_{Y}^{p_{2}}+\left\|\chi_{I_{N}} \varphi F_{N}\right\|_{Y}^{p_{2}}\right) \\
& \geq d_{1}^{-p_{2}}\left((\lambda \varepsilon)^{p_{2}}\left\|\nu_{0} f_{0}\right\|_{X}^{p_{2}}+\sum_{k=1}^{N-1}\left\|\chi_{I_{k}} \varphi\left(\nu_{k} F_{k}+\mu_{k}\right)\right\|_{Y}^{p_{2}}+(\lambda \varepsilon)^{p_{2}}\left\|\nu_{N} f_{N}\right\|_{X}^{p_{2}}\right) \\
& \geq d_{1}^{-p_{2}}\left((\lambda \varepsilon)^{p_{2}}\left\|\nu_{0} f_{0}\right\|_{X}^{p_{2}}\right. \\
& \left.+\left(\frac{1}{2,02}\right)^{p_{2}} \sum_{k=1}^{N-1}\left(\left|\nu_{k}\right|\left\|\chi_{I_{k}} \varphi\left(F_{k}-F_{k, I_{k}}\right)\right\|_{Y}\right)^{p_{2}}+(\lambda \varepsilon)^{p_{2}}\left\|\nu_{N} f_{N}\right\|_{X}^{p_{2}}\right) \\
& \geq d_{1}^{-p_{2}}\left((\lambda \varepsilon)^{p_{2}}\left\|\nu_{0} f_{0}\right\|_{X}^{p_{2}}\right. \\
& \left.+\left(\frac{\lambda \varepsilon}{2,02}\right)^{p_{2}} \sum_{k=1}^{N-1}\left(\left|\nu_{k}\right|\left\|f_{k}\right\|_{X}\right)^{p_{2}}+(\lambda \varepsilon)^{p_{2}}\left\|\nu_{N} f_{N}\right\|_{X}^{p_{2}}\right) \\
& =d_{1}^{-p_{2}}\left((\lambda \varepsilon)^{p_{2}}\left\|\nu_{0} f_{0}\right\|_{X}^{p_{2}}+\left(\frac{\lambda \varepsilon}{2,02}\right)^{p_{2}} \sum_{k=1}^{N-1}\|\| \nu_{k} f_{k}\left\|_{X}^{p_{2}}+(\lambda \varepsilon)^{p_{2}}\right\| \nu_{N} f_{N} \|_{X}^{p_{2}}\right) \\
& \geq\left(\frac{\lambda \varepsilon}{2,02 d_{1}}\right)^{p_{2}} \sum_{k=0}^{N}\left\|\nu_{k} f_{k}\right\|_{X}^{p_{2}} \\
& \geq\left(\frac{\lambda \varepsilon}{2,02 d_{1}}\right)^{p_{2}}\left(\sum_{k=0}^{N}\left\|\nu_{k} f_{k}\right\|_{X}^{p_{1}}\right)^{p_{1} / p_{2}}(N+1)^{1-p_{2} / p_{1}} \\
& \geq\left(\frac{\lambda \varepsilon}{2,02 d_{1} d_{2}}\right)^{p_{2}}(N+1)^{1-p_{2} / p_{1}}\|f\|_{X}^{p_{2}}
\end{aligned}
$$

Thus,

$$
a_{N}(H) \geq \frac{1}{2,02} d_{1}^{-1} d_{2}^{-1} \lambda \varepsilon(N+1)^{1 / p_{2}-1 / p_{1}},
$$

and, letting $\lambda \rightarrow 1$ the required lower bound follows. 
Remark 6. For Lebesgue spaces Theorems 6 and 7 were proved in [5], the extension to Lorentz spaces was given in [11].

\section{References}

1. C. Bennett And R. Sharpley, "Interpolation of operators," Pure Appl. Math. 129, Academic Press, 1988.

2. E. I. BerezhnoI, Weighted inequalities of Hardy type in general ideal spaces, Soviet Math. Dokl. 43 (1991), 492-495.

3. E. I. Berezhnoi, Sharp estimates of operators on the cones of ideal spaces, Proc. Steklov Inst. Math. 3 (1994), 3-34.

4. D. E. EDmunds AND W. D. Evans, "Spectral theory and differential operators," Oxford Univ. Press, 1987.

5. D. E. Edmunds, W. D. Evans And D. J. Harris, Approximation numbers of certain Volterra integral operators, J. London Math. Soc. 37 (1988), 471-489.

6. D. E. Edmunds, P. Gurka And L. Pick, Compactness of Hardy type integral operators in weighted Banach function spaces, Studia Math. 109 (1994), 73-90.

7. D. E. Edmunds And V. D. Stepanov, The measure of non-compactness and approximation numbers of certain Volterra integral operators, Math. Ann. 298 (1994), 41-66.

8. H. KÖNIG, "Eigenvalue distribution of compact operators," Birkhäuser, Boston, 1986.

9. L. V. Kantorovich And G. P. Akilov, "Functional Analysis," Pergamon, Oxford, 1982.

10. M. A. Krasnosel'ski And YA. B. Ruticki, "Convex function and Orlicz cpases," Noordhoff, Groningen, 1961.

11. E. Lomakina and V. Stepanov, On the compactness and approximation numbers of Hardy type integral operators in Lorentz spaces, J. London Math. Soc. 53(2) (1996), 369-382.

12. J. MARTÍN-REyes AND E. SAWYER, Weighted inequalities for Reimann-Liouville fractional integrals of order one and greater, Proc. Amer. Math. Soc. 106 (1989), 727-733.

13. R. Oinarov, Weighted inequalities for a class of integral operators, Soviet Math. Dokl. 44 (1992), 291-293.

14. R. Oinarov, Two-sided norm estimates for certain classes of integral operators, Proc. Steklov Inst. Math. 3 (1994), 205-214. 
15. B. OpIC And A. Kufner, "Hardy-type Inequalities," Pitman Research Notes in Math. Series 219, Longman SciTech., Harlow, 1990.

16. A. PIETsch, "Operator ideals," VEB Deutscher Verlag Wiss., Berlin, 1978.

17. E. T. SAWYER, Weighted Lebesgue and Lorentz norm inequalities for the Hardy operator, Trans. Amer. Math. Soc. 281 (1984), 329-337.

18. V. D. Stepanov, Weighted inequalities for a class of Volterra convolution operators, J. London Math. Soc. 45 (1992), 232-242.

19. V. D. Stepanov, Weighted norm inequalities for integral operators and related topics, in "Nonlinear Analysis, Function spaces and Applications," vol. 5, Olimpia Press, Prague, 1994, pp. 139-176.

\author{
Elena Lomakina: \\ Department of Mathematics \\ Khabarovsk State University \\ Tichookeanskaya 136 \\ Khabarovsk 680035 \\ RUSSIA
}

\author{
Vladimir Stepanov: \\ Computer Center \\ Far-Eastern Branch \\ of the Russian Academy of Sciences \\ Shelest 118-205 \\ Khabarovsk 680042 \\ RUSSIA
}

e-mail: 1600@as.khabarovsk.su

Primera versió rebuda el 8 d'abril de 1997 , darrera versió rebuda el 24 de febrer de 1998 